\title{
Relationship between ischemia modified albumin and lipid profile in type 2 diabetes mellitus
}

\author{
Priya Patil ${ }^{1, *}$, Shruthi Rai P $^{2}$ \\ ${ }^{1}$ Tutor, Dept. of Biochemistry, Belagavi Institute of Medical Sciences, Belgaum, Karnataka, ${ }^{2}$ Assistant Professor, Dept. of \\ Biochemistry, K.V.G. Medical College \& Hospital, Sullia, Karnataka, India \\ *Corresponding Author: \\ Email: dr.priyamallikarjun30@gmail.com
}

Received: $8^{\text {th }}$ February, 2018

Accepted: $21^{\text {st }}$ March, 2018

\begin{abstract}
Introduction: Ischemia modified albumin (IMA), is new biomarker of oxidative stress and ischemia. IMA being increased in Diabetes Mellitus has been described in many studies. Diabetes Mellitus (DM) a metabolic disorder is characterised by hyperglycemia, hypertension and hyperlipidemia. So, our aim was to estimate the levels of IMA in type 2 DM subjects. Also to evaluate the relationship of IMA with all the parameters in lipid profile (TC, LDL, HDL, TG) in type 2 DM subjects.

Materials and Methods: 120 subjects were selected from the medicine OPD of Justice K S Hegde Charitable Hospital, Mangalore for study. The subjects attending the hospital were from coastal areas of southern India. Out of which 60 subjects were type $2 \mathrm{DM}$ patients as per the ADA guidelines and 60 subjects were age and sex matched controls. The biochemical parameters estimated were FPS, 2hr-PG, HbA1C, Lipid profile (TC, TG, LDL, VLDL, HDL) and IMA. The results were expressed in absorbance units (ABSU). Data obtained was analyzed using SPSS 20.

Results: The Fasting and postprandial plasma glucose, HbA1c, serum TG, TC, LDL, HDL, IMA were significantly increased in type 2 DM patients than in control subjects with the $\mathrm{p}$ value $<0.001$. A significant correlation was found between Lipid profile and IMA.

Conclusion: The present study suggests that measuring IMA in dyslipidemic diabetic patients will provide an early index of ischemia and its severity. This would help in the better management and prognosis of DM.
\end{abstract}

Keywords: Hyperglycemia, Hyperlipidemia, Type 2 Diabetes mellitus, IMA.

\section{Introduction}

Ischemia modified albumin (IMA), is one of the recent biomarker of oxidative stress and ischemia. ${ }^{1}$ Ischemia through its pathophysiological events like hypoxia and free oxygen radical causes conformational changes in the $\mathrm{N}$-terminal end of albumin resulting in ischemia modified albumin. ${ }^{2}$ IMA levels are raised in many clinical conditions like myocardial ischemia, thalassemia, ovarian torsion, chronic renal failure, hypothyroidism and malignancy. IMA being increased in diabetes mellitus has been described in many studies. Kaefer et al., found a correlation between C-reactive protein, hyperglycemia, and glycated albumin in type 2 diabetes subjects. ${ }^{3}$

DM is a group of metabolic disorder which is characterized by hyperglycemia, hypertension and hyperlipidemia are the independent accelerating factors of hyperglycemia and tissue damage. This due to repeated exposure will lead to macro and microvascular complications. ${ }^{4}$

The pathological changes resulting from hyperglycemia include capillary basement membrane thickening, deposition and oxidation of cholesterol and cellular proliferation resulting in narrowing of arterial vessels. ${ }^{5}$ Endothelial dysfunction along with low grade systemic inflammation are the initiating factors of atherosclerosis. The diagnosis of overt diabetes is usually made after the onset of macrovascular complications like stroke, myocardial infarction and peripheral arterial diseases. ${ }^{6}$

In view of the above finding our aim is to estimate the levels of IMA in type 2 DM subjects and evaluate its relationship with all the parameters in lipid profile (TC, LDL, HDL, TG) in T2DM subjects. If relationship is found IMA could be used as a parameter for early identification of the occurrence of the cardiovascular complication in T2DM subjects.

\section{Materials and Methods}

120 subjects were selected from the medicine OPD of Justice K S Hegde Charitable Hospital, Mangalore for the study. The study subjects were from coastal areas of southern India like Sulia, Puttur, Beltangadi, Mangalore and Kasargod aged >40years. Out of which 60 were diagnosed with T2DM as per the American Diabetes Association (ADA) 2016 guidelines $^{7}$ and remaining 60 were age and sex matched controls. The ethical clearance was obtained, a detailed history and written informed consent was taken from all the subjects. Subjects with $\mathrm{h} / \mathrm{o}$ ischemic events, liver and kidney dysfunction, infection, corticosteroid therapy, pregnancy were excluded from the study.

Six $\mathrm{ml}$ of overnight fasting venous blood sample was collected from each subject under aseptic conditions, from the antecubital vein. $1.5 \mathrm{ml}$ was collected into fluoride tube, $1.5 \mathrm{ml}$ into EDTA tube and remaining into plain vacutainer. Then $2.0 \mathrm{ml}$ of $2 \mathrm{hr}$ - 
postprandial blood sample was collected in fluoride tube for 2hr-PG estimation. All the tubes were subjected for centrifugation at $3000 \mathrm{rpm}$ for $3 \mathrm{~min}$. Plasma in fluoride tube was used for the estimation of glucose. The EDTA tube sample was used for HbA1c estimation. approximately $0.5 \mathrm{ml}$ of the serum separated from the blood in plain vacutainer was transferred into small eppendorf tube and stored at $-20^{\circ} \mathrm{C}$ until analysis for IMA estimation.

The rest of the serum was used for estimation of the total cholesterol (TC), triglyceride (TG) and high density lipoprotein (HDL). All estimations were performed in COBAS C311 autoanalyzer. The low density lipoprotein (LDL) was calculated using Friedwald Formula. ${ }^{8}$

Serum IMA was determined manually by the albumin cobalt binding assay, the method described by Bar-Or et al. ${ }^{9}$ The results were given in absorbance units (ABSU).
Data obtained was analyzed using SPSS 20. All the results were expressed in mean \pm SD. For parametric data, students t- test was used to compare between two independent mean groups. For correlation studies, Karl Pearson Correlation was used. The Value of $\mathrm{p}<0.05$ was considered to be statistically significant.

\section{Results}

120 subjects included in the study were divided into two groups. 60 were diabetic cases and remaining 60 were controls. Among the diabetic cases 35 were females and 25 were males. And among the control group 32 were females and 28 were males. The mean age of the female diabetic cases were $58.50 \pm 12.41$ years and male diabetic cases was $56.36 \pm 10.31$ years. The mean duration of T2DM among the males was $11.68 \pm 7.36$ and females were $12.70 \pm 8.02$. The duration of diabetes was almost same between the male and female diabetic subjects. No statistical difference was noted.

Table 1: Shows the demographic details of all subjects involved in the study

\begin{tabular}{|l|c|c|c|c|}
\hline \multirow{2}{*}{ Parameters (mean \pm SD) } & \multicolumn{2}{|c|}{ Diabetic cases (60) } & \multicolumn{2}{c|}{ Controls (60) } \\
\cline { 2 - 5 } & Male (25) & Female(35) & Male (28) & Female (32) \\
\hline Age (years) & $56.36 \pm 10.31$ & $58.50 \pm 12.41$ & $56.78 \pm 11.09$ & $55.73 \pm 13.43$ \\
\hline Duration of diabetes(years) & $11.68 \pm 7.36$ & $12.70 \pm 8.02$ & 0 & 0 \\
\hline
\end{tabular}

Table 2 shows the laboratory findings of all the subjects included in the study. The Fasting and postprandial plasma glucose, HbA1c, serum TG, TC,
LDL, HDL, IMA were significantly increased in T2DM patients than in control subjects with the $p$ value $<0.001$ except for VLDL with the P value $<0.05$.

Table 2: Shows the laboratory finding of the subjects

\begin{tabular}{|l|c|c|c|}
\hline \multicolumn{1}{|c|}{ Parameters (mean \pm SD) } & Diabetic Cases (60) & Controls (60) & P-value \\
\hline Fasting plasma glucose(mg/dl) & $180.7 \pm 57.35$ & $94.53 \pm 3.48$ & $<0.001$ \\
\hline 2-hr postprandial plasma glucose (mg/dl) & $296.60 \pm 88.8$ & $166 \pm 10.25$ & $<0.001$ \\
\hline HbA1c (\%) & $8.5 \pm 1.9$ & $5.2 \pm 0.2$ & $<0.001$ \\
\hline Serum triglycerides(mg/dl) & $253 \pm 18$ & $140 \pm 12.1$ & $<0.001$ \\
\hline Serum total cholesterol(mg/dl) & $262 \pm 19.1$ & $150 \pm 19$ & $<0.001$ \\
\hline Serum VLDL(mg/dl) & $46 \pm 5.1$ & $29 \pm 2.6$ & $<0.05$ \\
\hline Serum LDL (mg/dl) & $159 \pm 22.3$ & $89 \pm 7.4$ & $<0.001$ \\
\hline Serum HDL (mg/dl) & $29 \pm 3.9$ & $55 \pm 4.5$ & $<0.001$ \\
\hline Serum IMA (ABSU) & $0.759 \pm 0.17$ & $0.269 \pm 0.10$ & $<0.001$ \\
\hline
\end{tabular}

Table 3 shows the correlation of IMA with the parameters of lipid profile. The Fasting and postprandial plasma glucose, $\mathrm{HbA1c}$, serum TG, TC,
LDL, HDL, IMA were are significantly increased in T2DM patients than in control subjects with the $\mathrm{p}$ value $<0.001$

Table 3: Correlation of IMA with parameters of Lipid profile

\begin{tabular}{|l|c|c|}
\hline \multirow{2}{*}{ Lipid profile parameters } & \multicolumn{2}{|c|}{ Serum IMA } \\
\cline { 2 - 3 } & r-value & p-value \\
\hline Serum Triglycerides & 0.56 & $<0.05$ \\
\hline Serum cholesterol & 0.67 & $<0.05$ \\
\hline Serum VLDL & 0.61 & $<0.05$ \\
\hline Serum LDL & 0.80 & $<0.001$ \\
\hline Serum HDL & 0.78 & $<0.001$ \\
\hline
\end{tabular}




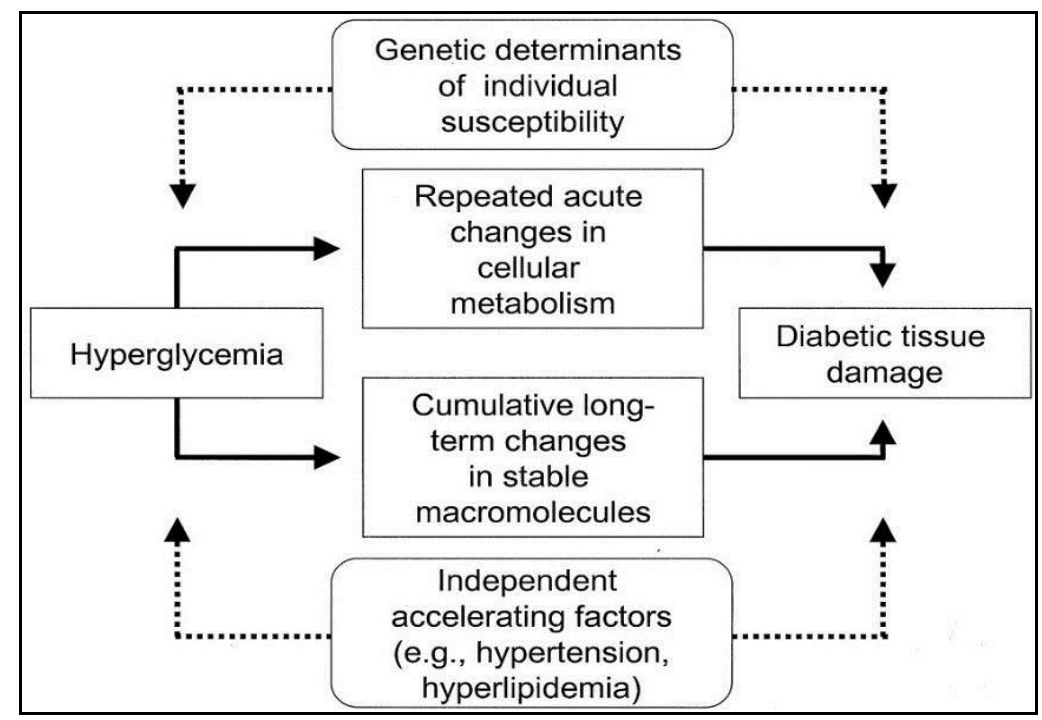

Fig. 1: Hyperglycaemia causing tissue damage

[Ref: Michael Brownlee Diabetes 2005; 54: 1615-1625@2005 by ADA]

\section{Discussion}

It has been observed that occurrence of type $2 \mathrm{DM}$ at a much younger age is becoming more common ${ }^{10}$. Therefore its early diagnosis and prevention of the complications like myocardial infarction and stroke in younger subjects is important. Hyperglycemia increases oxidative stress by many pathways. An important one is the generation of superoxide radicals via electron transport chain. ${ }^{11}$ These free radicals not only damage the endothelial lining of the vessels and lead to macrovascular and microvascular complications but also cause the alteration in the structure of circulating albumin. Therefore results in increased production of IMA in the presence of oxidative stress.

In this study we have evaluated the levels of IMA and Lipid profile in type $2 \mathrm{DM}$ patients and in control subjects. The study showed that the IMA and lipid profile were significantly elevated in type 2 DM subjects. A significant positive correlation was found between the IMA and the lipid profile. Our finding were in agreement with the study done by Ukrinc et $\mathrm{al}^{12}$ who found significantly increased levels of IMA in patients with T2DM with or without cardiovascular complications than controls. Kaefer et $\mathrm{al}^{3}$ showed the increased levels of triglycerides and IMA in type 2 DM patients than in healthy controls. Rajendra et al also found the increased levels of IMA in diabetes patients with peripheral neuropathy than without peripheral neuropathy. ${ }^{13}$ Turk et al described the role of IMA in the diabetic nephropathy. ${ }^{14}$ IMA could thus serve as an early indicator of glycaemic control, severity of complications and disease progression. Another study revealed the higher levels of triglycerides, TC, LDL and IMA in type $2 \mathrm{DM}$ patients with peripheral arterial diseases than those without peripheral arterial diseases. ${ }^{15}$

\section{Conclusion}

The present study suggests that measuring IMA in dyslipidemic diabetic patients would provide an early index of ischemia and its severity. This will help in the better management and prognosis of diabetes mellitus.

\section{References}

1. M Idris, S Kurban, F Humeyra, P Yerlikaya, H Polat. Obesity is an independent determinant of ischemia modified albumin. Available from http://www.karger.com/Article/Pdf/34/3954pdf, accessed $15^{\text {th }}$ may 2015.

2. WS Aronow, C Ahn, MB Weiss, S Babu. Relation of increased hemoglobin a (1c) levels to severity of peripheral arterial disease in patients with diabetes mellitus. American Journal of Cardiology. 2007;99:146869.

3. M Kaefer, S Piva J, JAM De-Carvalho, DB Da Silva, AM Becker, AC Coelho et al. Association between ischemia modified albumin, inflammation and hyperglycemia in type 2 diabetes mellitus. Clinical Biochemistry. 2010;43(4-5):450-54.

4. Michael Brownlee. The Pathobiology of Diabetic Complications. Diabetes 2005;54:1615-1625. https://doi.org/10.2337/diabetes.54.6.1615

5. Dash PMM. Ischemia modified albumin - An indicator of widespread endothelial damage in diabetes mellitus. Journal of Physiobiochemical Metabolism. 2014;03(01):1-4.

6. American Diabetes Association. Diagnosis and Classification of diabetes mellitus. Diabetes care. 2012;35(suppl1):S64-71. https://doi.org/10.2337/dc12s064

7. Libby P, Ridker PM. Novel inflammatory markers of coronary risk: theory versus practice. Circulation. 1999;100(11): 1148-50.

8. American diabetes association. Standards of medical care in diabetes-2009. Diabetes care 2009;32:513-61.

9. Friewald, W.T, R.I. Levy and D.S. Frederickson, 1972. Estimation of the concentration of low density lipoprotein cholesterol in plasma without use of the preparative ultracentrifuge. Clin Chem, 1972 Jun;18(6):499-502. 
10. D Bar-Or, E Lau, JV Winkler. A novel assay for cobaltalbumin binding and its potential as a marker for myocardial ischemia- a preliminary report. J Emerg Med. 2000 Nov;19(4):311-315.

11. Wild Sarah, Roglic Gojka, Green Anders et al. Global Prevalence of Diabetes: Estimates for the year 2000 and projections for 2030. Diabetes Care. 2004;27:1047-1053.

12. Rolo, A.P. and C.M. Plamia, 2006. Diabetes and mitochondrial function: role of hyperglycemia and oxidative stress. Toxicol Appl Pharmacol., 2006 Apr 15;212(2):167-78.

13. Ukrinc, K, S. Eminagaoglu, H.O. Eroz, C. Erem, C. Karahan, A.B. Hacihasanoglu and M. Kocak, 2009. A novel indicator of widespread endothelial damage and ischemia in diabetic patients. Endocrine. 2009 Dec;36(3):425-32.

14. Chawla $\mathrm{R}$ et al. Ischemia modified albumin (IMA)- A marker of Glycemic control and vascular complications in type 2 diabetes mellitus. J Clin Diagn Res. 2016 Mar;10(3):BC13-6.
15. Turk A, Nuhoglu I, Mentese A, Karahan SC, Erdol H, Erem $\mathrm{C}$. The relationship between diabetic retinopathy and serum levels of ischemia modified albumin and Malondialdehyde. Retina. 2011;31(3):602-08.

16. Ma, S.G., C.L. Wei, B. Hong and W.N. Yu, 2011. Ischemia modified albumin in type 2 diabetic patients with and without peripheral arterial disease. Clinics (Sao Paulo), 2011;66(10):1677-1680.

How to cite this article: Patil P, Rai SP. Relationship between ischemia modified albumin and lipid profile in type 2 diabetes mellitus. Int $\mathrm{J}$ Clin Biochem Res. 2018;5(3):473-476. 